\title{
Diversity of Tetracycline Resistance Genes in Bacteria Isolated from Various Agricultural Environments
}

\author{
YURI KOBASHI $^{1 *}$, AKIRA HASEBE ${ }^{2 \dagger}$, MichINORI NiSHIO ${ }^{3}$ and HIROO UCHIYAMA ${ }^{1}$ \\ ${ }^{1}$ Graduate School of Life and Environmental Sciences, University of Tsukuba, 1-1-1, Tennodai, Tsukuba, \\ Ibaraki 305-8572, Japan \\ ${ }^{2}$ National Institute for Agro-Environmental Sciences, 3-1-3, Kannondai, Tsukuba, Ibaraki 305-8604, Japan \\ ${ }^{3}$ Formerly of the Institute of Agricultural and Forest Engineering, University of Tsukuba, 1-1-1, Tennodai, \\ Tsukuba, Ibaraki 305-8572, Japan
}

(Received September 11, 2006-Accepted November 22, 2006)

A total of 350 tetracycline-resistant $\left(\mathrm{Tc}^{\mathrm{r}}\right)$ bacteria were isolated from livestock feces, farmyard manure (FYM), and soil in Japan. The isolates were classified into 28 established genera of the phyla Actinobacteria, Bacteriodetes, Firmicutes and Proteobacteria by studying partial 16S rRNA gene sequences. PCR analysis revealed that 249 of the $350 \mathrm{Tc}^{\mathrm{r}}$ isolates contained at least 1 of $15 \mathrm{Tc}^{\mathrm{r}}$ genes (tet genes): 140 isolates contained an efflux pump gene and 109 isolates contained a ribosomal protection protein (RPP) gene. The predominant fecal isolates were identified as members of the genera Enterococcus and Escherichia, carrying tet $(\mathrm{M})$ and tet $(\mathrm{H})$, respectively. The predominant soil isolates were identified as members of the genera Burkholderia and Afipia, carrying tet $(\mathrm{Z})$ and tet(B), respectively. This study is the first to describe the presence of tet genes in members of the genera Bordetella, Bradyrhizobium, Burkholderia, Dyella, Flexibacter, Kurthia, Luteibacter, Lysobacter, "Nordella", Ochrobactrum, Pediococcus, Rhodopseudomonas, and Vagococcus. It has revealed that tet genes are distributed in much more diverse phylogenetic groups of bacteria in the environment than has been reported previously.

Key words: farmyard manure, livestock feces, soil, tetracycline-resistant bacteria, tet gene

An important issue with antibiotic resistance is the fact that the widespread use of antibiotics not only selects for drug-resistant pathogenic bacteria, but also exerts selective pressure on the normal commensal microbiota. It is thought that, in light of the ubiquitously demonstrated phenomenon of horizontal transfer of antibiotic resistance genes in the microbial world, the presence of such reservoirs may explain the rapid dissemination of antibiotic resistance from commensal organisms to pathogenic microbiota ${ }^{3)}$.

Animal manure contains high numbers of resistant bacteria $^{6,22)}$. Since animal manure is frequently spread on farmland, a risk exists not only for the spread of resistant

\footnotetext{
* Corresponding author. E-mail address: kobashi@affrc.go.jp; Tel.: +81-29-838-8648; Fax: +81-29-838-8648.

$\uparrow$ Present address: Agriculture, Forestry and Fisheries Research Council Secretariat, Ministry of Agriculture, Forestry and Fisheries of Japan, 1-2-1, Kasumigaseki, Chiyoda, Tokyo 100-8950, Japan
}

bacteria, but also for horizontal transfer of resistance genes to indigenous bacteria ${ }^{1)}$. In a recent study, soil bacteria isolated from soil and groundwater underlying swine production facilities contained tetracycline resistance genes that are identical to those found in bacteria isolated from manure ${ }^{7)}$.

In our previous work, we examined the distribution of antibiotic-resistant bacteria in animal-soil systems by analyzing 19 samples of livestock feces, solid farmyard manure (FYM), soil from arable fields and orchards treated with FYM or not, and forest soils ${ }^{12}$. In the fecal samples from livestock fed with antibiotics as feed additives, a high occurrence of antibiotic-resistant bacteria was observed. In contrast, in the 2 fecal samples from livestock fed without antibiotics as feed additives, a relatively low occurrence of antibiotic-resistant bacteria was observed. The results suggested that there might be a link between the use of in-feed antibiotics and the prevalence of antibiotic-resistant bacteria 
in animal feces. Furthermore, our study also showed that the occurrence of broad-range multi-drug resistance (MDR) was much higher in bacteria in the feces of swine fed with antibiotics than in bacteria in the natural forest soil, suggesting that the occurrence of broad-range MDR in the feces was enhanced by the use of antibiotics as feed additives.

Tetracyclines are broad-spectrum antimicrobial agents, exhibiting activity against a wide range of Gram-positive and Gram-negative bacteria, protozoan parasites, and atypical organisms such as chlamydiae, mycoplasmas, and rickettsiae. The favorable antimicrobial properties of these agents and the absence of major adverse side effects have led to their extensive use in treating infections in humans, farmed fishes, and animals ${ }^{9,16,18)}$. In many countries, tetracyclines are added at subtherapeutic levels to animal feeds as growth promoters. As a result, tetracycline resistance genes have spread widely in clinical and natural environments ${ }^{2-7}$, and tetracycline-resistant bacteria, particularly those isolated from farming environments, show broad-range $\mathrm{MDR}^{12)}$. Although several studies have examined tetracycline resistance among bacteria, most have employed pathogenic and important epidemiologic isolates ${ }^{5)}$. These studies, while useful, did not give enough information about the presence and the types of tet genes that are present naturally in the environment.

In the present study, for a better understanding of the resistance genes and their distribution in the environment, a total of 350 tetracycline-resistant bacterial isolates from 19 environmental samples were used. We also investigated the distribution of tet genes (11 genes encoding tetracycline efflux pumps: tet( $\mathrm{A}), \operatorname{tet}(\mathrm{B}), \operatorname{tet}(\mathrm{C}), \operatorname{tet}(\mathrm{D}), \operatorname{tet}(\mathrm{E}), \operatorname{tet}(\mathrm{G})$, $\operatorname{tet}(\mathrm{H}), \operatorname{tet}(\mathrm{J}), \operatorname{tet}(\mathrm{Y}), \operatorname{tet}(\mathrm{Z})$, and $\operatorname{tet}(30)$; and 8 genes encoding ribosomal protection proteins $(\mathrm{RPP})$ : tet $(\mathrm{M})$, tet $(\mathrm{S})$, $\operatorname{tet}(\mathrm{O}), \operatorname{tet}(\mathrm{W}), \operatorname{tet}(\mathrm{Q}), \operatorname{tet}(\mathrm{T}), \operatorname{tet} \mathrm{P}(\mathrm{B})$, and $\operatorname{otr}(\mathrm{A}))$.

\section{Materials and Methods}

\section{Isolation of $\mathrm{Tc}^{r}$ bacteria}

In order to characterize $\mathrm{Tc}^{\mathrm{r}}$ bacteria from various sources, $\mathrm{Tc}^{\mathrm{r}}$ isolates were obtained from 6 fecal samples, $6 \mathrm{FYM}$ samples, and 7 soil samples in Japan. Sources and preparation of the samples were described previously ${ }^{12)}$. To isolate $\mathrm{Tc}^{\mathrm{r}}$ bacteria from fecal samples, the following 4 types of agar media each containing $50 \mu \mathrm{g} \mathrm{ml}^{-1}$ of tetracycline (Sigma Chemical Co, St. Louis, MO, USA) were used: PTYG agar medium for detection of aerobic heterotrophic tetracycline resistant bacteria ${ }^{12)}$; EF agar medium (Nissui, Tokyo, Japan) for detection of tetracycline resistant enterococci; MRS agar medium (Becton, Dickinson and Co.,
Franklin Lakes, NJ, USA) for detection of tetracycline resistant lactic acid bacteria; and Desoxycolate agar medium (Nissui) for detection of tetracycline resistant coliform bacteria. To isolate $\mathrm{Tc}^{\mathrm{r}}$ bacteria from FYM samples, PTYG and Desoxycolate agar media each containing $50 \mu \mathrm{g} \mathrm{ml}^{-1}$ of tetracycline were used. To isolate $\mathrm{Tc}^{\mathrm{r}}$ bacteria from soil samples, PTYG agar medium containing $50 \mu \mathrm{g}$ $\mathrm{ml}^{-1}$ of tetracycline was used.

We used the dilution plate method to isolate $\mathrm{Tc}^{\mathrm{r}}$ bacteria by making 10-fold dilutions with phosphate-buffered saline (pH 7.0). PTYG agar plates were incubated at $30^{\circ} \mathrm{C}$ for 7 days, EF agar plates and MRS agar plates were incubated at $37^{\circ} \mathrm{C}$ for $48 \mathrm{~h}$, and Desoxycolate agar plates were incubated at $37^{\circ} \mathrm{C}$ for $24 \mathrm{~h}$. After the incubation period, about ten bacterial strains were selected randomly. Purification of the bacterial isolates was carried out using PTYG agar medium or Luria-Bertani agar medium ${ }^{13}$ ) containing $50 \mu \mathrm{g} \mathrm{ml}^{-1}$ of tetracycline (Sigma).

\section{Characterization of the Tcr bacterial isolates}

Template DNA was extracted from the $\mathrm{Tc}^{\mathrm{r}}$ isolates by the hexadecyltrimethylammonium bromide (CTAB) method ${ }^{20)}$. PCR primers 63f (5'-CAGGCCTAACACATGCAAGTC$\left.3^{\prime}\right)$ and 1387 r (5'-GGGCGGWGTGTACAAGGC-3') ${ }^{15}$ ) were used to amplify the segment of the bacterial $16 \mathrm{~S}$ rRNA gene. PCR was carried out using a basic reaction mixture containing 1.0 U of Ex Taq polymerase (Takara, Shiga, Japan), $5 \mu$ l of $10 \times \mathrm{Ex}$ Taq reaction buffer (Takara), 10 nmol of each deoxynucleoside triphosphate, $1 \mathrm{nmol}$ of template DNA, and $25 \mathrm{pmol}$ of each primer in a total volume of $50 \mu \mathrm{l}$. PCR amplification was conducted in an I-Cycler thermal cycler (Bio Rad, Hercules, CA, USA) for 30 cycles, each consisting of $30 \mathrm{sec}$ at $94^{\circ} \mathrm{C}, 30 \mathrm{sec}$ at $58^{\circ} \mathrm{C}$, and $30 \mathrm{sec}$ at $72^{\circ} \mathrm{C}$, with a final extension for $10 \mathrm{~min}$ at $72^{\circ} \mathrm{C}$. The PCR products were purified with the Ultra Clean PCR Clean-up DNA Purification Kit (Mo Bio Laboratories, Solana Beach, CA, USA). For the restriction fragment length polymorphism (RFLP) analysis ${ }^{17}$, the PCR products were digested with endonucleases HaeIII and RsaI (Toyobo, Osaka, Japan). Digested DNA fragments were analyzed by horizontal electrophoresis on $2.0 \%$ agarose gel in TAE ( $40 \mathrm{mM}$ Tris, $20 \mathrm{mM}$ acetate, and $2 \mathrm{mM}$ EDTA) and staining with ethidium bromide. RFLP analysis was conducted for 350 isolates, which were classified into 69 groups on the basis of the band patterns. Then, direct sequencing of approximately $1,200 \mathrm{bp}$ of PCR products of representative isolates from each of the 69 groups was performed with a BigDye Terminator v3.1 cycle sequencing kit (Applied Biosystems, Foster City, CA, USA). The reaction products were analyzed with 
an ABI PRISM 3730 Genetic Analyzer (Applied Biosystems). The phylogenic positions of the isolates were determined by searching the DDBJ database (http:// www.ddbj.nig.ac.jp/Welcome-e.html) with the BLAST program.

The sequences of bacterial strains generated in this study have been deposited in the DDBJ database under accession numbers AB272318 to AB272386.

\section{PCR detection of tet genes}

All isolates were tested for the presence of tet genes by PCR. The primers used for PCR amplification of the 19 tet genes were described by Aminov et al. ${ }^{3,4)}$. The target tet genes were 11 genes encoding tetracycline efflux pumps: $t e t(\mathrm{~A}), \operatorname{tet}(\mathrm{B}), \operatorname{tet}(\mathrm{C}), \operatorname{tet}(\mathrm{D}), \operatorname{tet}(\mathrm{E}), \operatorname{tet}(\mathrm{G}), \operatorname{tet}(\mathrm{H}), \operatorname{tet}(\mathrm{J})$, $\operatorname{tet}(\mathrm{Y})$, tet $(\mathrm{Z})$, and tet $(30)$; and 8 genes encoding ribosomal protection proteins $(\mathrm{RPP}): \operatorname{tet}(\mathrm{M}), \operatorname{tet}(\mathrm{S}), \operatorname{tet}(\mathrm{O}), \operatorname{tet}(\mathrm{W})$,
$\operatorname{tet}(\mathrm{Q}), \operatorname{tet}(\mathrm{T}), \operatorname{tet} \mathrm{P}(\mathrm{B})$, and $\operatorname{otr}(\mathrm{A})$.

All PCRs for detecting the presence or absence of resistance genes were based on the method described by Aminov et $a .^{3,4)}$. The validity of the PCR system and the sizes of amplification products were ascertained by using positive control plasmids each carrying the tet gene ${ }^{3,4}$. Nucleotide sequences of the PCR products were determined with a BigDye Terminator v3.1 cycle sequencing kit (Applied Biosystems).

\section{Results}

\section{Isolation of bacteria resistant to tetracycline}

A total of $350 \mathrm{Tc}^{\mathrm{r}}$ bacterial isolates were obtained from the various sources on the 4 types of agar media (Table 1).

RFLP analysis was conducted for each of the 350 isolates, which were subsequently classified into 69 groups on

Table 1. Tetracycline-resistant isolates, their origins, and types of tet gene

\begin{tabular}{|c|c|c|c|c|c|}
\hline \multirow{2}{*}{ Origin } & \multirow{2}{*}{ Media $^{\mathrm{a}}$} & \multirow{2}{*}{ No. of isolates } & \multicolumn{3}{|c|}{ tet genes } \\
\hline & & & Efflux genes & RPP genes & Unknown \\
\hline \multirow[t]{4}{*}{ Swine feces } & PTYG & 25 & 10 & 6 & 9 \\
\hline & Desoxycolate & 28 & 20 & 4 & 4 \\
\hline & $\mathrm{EF}$ & 21 & 1 & 20 & 0 \\
\hline & Subtotal & 74 & 31 & 30 & 13 \\
\hline \multirow[t]{5}{*}{ Poultry feces } & PTYG & 28 & 22 & 5 & 1 \\
\hline & Desoxycolate & 26 & 17 & 6 & 3 \\
\hline & $\mathrm{EF}$ & 30 & 0 & 30 & 0 \\
\hline & MRS & 18 & 6 & 10 & 2 \\
\hline & Subtotal & 102 & 45 & 51 & 6 \\
\hline \multirow[t]{3}{*}{ Swine FYM } & PTYG & 18 & 4 & 7 & 7 \\
\hline & Desoxycolate & 3 & 3 & 0 & 0 \\
\hline & Subtotal & 21 & 7 & 7 & 7 \\
\hline \multirow[t]{3}{*}{ Poultry FYM } & PTYG & 13 & 2 & 2 & 9 \\
\hline & Desoxycolate & 10 & 2 & 4 & 4 \\
\hline & Subtotal & 23 & 4 & 6 & 13 \\
\hline \multirow[t]{3}{*}{ Cattle FYM } & PTYG & 10 & 8 & 2 & 0 \\
\hline & Desoxycolate & 8 & 6 & 0 & 2 \\
\hline & Subtotal & 18 & 14 & 2 & 2 \\
\hline Soil (swine FYM applied) & PTYG & 9 & 2 & 3 & 4 \\
\hline Soil (poultry FYM applied) & PTYG & 27 & 20 & 2 & 5 \\
\hline Soil (FYM not applied) & PTYG & 32 & 11 & 8 & 13 \\
\hline Forest soil & PTYG & 44 & 6 & 0 & 38 \\
\hline Total & All media & 350 & 140 & 109 & 101 \\
\hline
\end{tabular}

a PTYG: PTYG agar medium for detection of heterotrophic bacteria; Desoxycolatexycolate: Desoxycolate agar medium for detection of coliform bacteria; EF: EF agar medium for detection of enterococci; MRS: MRS agar medium for detection of lactic acid bacteria. 
the basis of different band patterns. Then, direct sequencing (approximately 1,200 bp) of PCR products of representative isolates from each of the 69 groups was performed. All of the sequence results showed more than $94.5 \%$ similarity to known genera, and all isolates were classified with the same member as the nearest genera.

The $350 \mathrm{Tc}^{\mathrm{r}}$ isolates affiliated to 28 bacterial genera (20 genera of Gram-negative and 8 genera of Gram-positive Tcr bacteria) (Table 2). From the 176 animal fecal isolates, 11 bacterial genera were classified. The predominant fecal isolates were identified as members of the genus Enterococcus to which 60 isolates belonged and the genus Escherichia to which 53 belonged. From the 62 FYM isolates, 9 bacterial genera were classified. The predominant FYM isolates were identified as members of the genus Serratia to which 16 isolates belonged. From the 102 soil isolates, 15 bacterial genera were classified. The predominant soil isolates were identified as members of the genus Burkholderia to which 47 isolates belonged.

\section{Distribution of tet genes}

\section{Outlines from all isolates}

The $350 \mathrm{Tc}^{\mathrm{r}}$ bacterial isolates were examined for the presence of 19 types of $\mathrm{Tc}^{\mathrm{r}}$ determinant. PCR analysis indicated that $71.1 \%$ of the isolates contained at least 1 of 15 of those $19 \mathrm{Tc}^{\mathrm{r}}$ genes. Among all $\mathrm{Tc}^{\mathrm{r}}$ bacterial isolates, tetracycline resistance genes encoding efflux pumps were detected in $40 \%$ (140 isolates), and those encoding RPP were detected in $31.1 \%$ (109 isolates) (Table 1).

\section{Isolates from animal feces}

In the swine feces, 31 isolates contained the efflux pump gene $\operatorname{tet}(\mathrm{A}), \operatorname{tet}(\mathrm{B}), \operatorname{tet}(\mathrm{C}), \operatorname{tet}(\mathrm{H}), \operatorname{tet}(\mathrm{J})$, or tet $(\mathrm{Y})$, and 30 isolates contained the RPP gene $\operatorname{tet}(\mathrm{M}), \operatorname{tet}(\mathrm{Q})$, or $\operatorname{tet}(\mathrm{W})$ (Tables 3 and 4). The most common tet gene identified was $\operatorname{tet}(\mathrm{M})$, which was found in $39.3 \%$ of all the isolates. Of the $24 \operatorname{tet}(\mathrm{M})$ positive isolates, 20 were identified as members of the genus Enterococcus.

In the poultry feces, 45 isolates contained the efflux pump gene tet $(\mathrm{A}), \operatorname{tet}(\mathrm{B}), \operatorname{tet}(\mathrm{C}), \operatorname{tet}(\mathrm{H})$, or tet $(\mathrm{J})$, and 51 isolates contained the RPP gene $\operatorname{tet}(\mathrm{M}), \operatorname{tet}(\mathrm{O}), \operatorname{tet}(\mathrm{Q})$, or tet $(\mathrm{W})$. The most common tet gene identified was tet $(\mathrm{M})$, which was found in $43.8 \%$ of all the isolates. Of the 42 tet $(\mathrm{M})$ positive isolates, 30 were identified as members of the genus Enterococcus. The second most common gene identified was tet $(\mathrm{H})$, which was found in $28.1 \%$ of all the isolates. Of the $29 \operatorname{tet}(\mathrm{H})$ positive isolates, 19 were identified as members of the genus Escherichia.

\section{Isolates from solid FYM}

In the swine FYM, 7 isolates contained the efflux pump gene tet $(\mathrm{B})$ or tet $(\mathrm{J})$, and 7 isolates contained the RPP gene $\operatorname{tet}(\mathrm{M})$, tet $(\mathrm{S})$, or $\operatorname{tet}(\mathrm{W})$ (Tables 3 and 4$)$. In the poultry FYM, 4 isolates contained the efflux pump gene tet $(\mathrm{B})$, and 6 isolates contained the RPP gene $\operatorname{tet}(\mathrm{S})$ or $\operatorname{tet}(\mathrm{W})$. In the cattle FYM, 14 isolates contained the efflux pump gene tet $(\mathrm{B})$, tet $(\mathrm{J})$, or $\operatorname{tet}(\mathrm{Z})$, and 2 isolates contained the RPP gene tet $(\mathrm{W})$.

\section{Isolates from soils}

In soil to which swine FYM had been applied, $2 \mathrm{Tc}^{\mathrm{r}}$ isolates carried efflux pump genes-1 carried tet $(\mathrm{B})$ and 1 carried $\operatorname{tet}(\mathrm{J})$-and 3 isolates contained the RPP gene $\operatorname{otr}(\mathrm{A})$ or $\operatorname{tet}(\mathrm{W})$ (Tables 3 and 4$)$. In soil to which poultry FYM had been applied, $20 \mathrm{Tc}^{\mathrm{r}}$ isolates carried the efflux pump gene $\operatorname{tet}(\mathrm{B}), \operatorname{tet}(\mathrm{G}), \operatorname{tet}(\mathrm{J})$, or tet $(\mathrm{Z})$, and 2 isolates contained the $\mathrm{RPP}$ gene tet $(\mathrm{Q})$ or tet $(\mathrm{W})$. The most common tet gene was tet(B), which was found in 9 isolates. In the soil to which FYM had not been applied, 11 isolates contained the efflux pump gene tet $(\mathrm{B}), \operatorname{tet}(\mathrm{G}), \operatorname{tet}(\mathrm{J})$, or $\operatorname{tet}(\mathrm{Z})$, and 8 isolates contained the RPP gene tet $(\mathrm{M}), \operatorname{otr}(\mathrm{A})$, or tet $(\mathrm{W})$. In the forest soil, all $6 \mathrm{Tc}^{\mathrm{r}}$ isolates carried efflux pump genes; no isolates contained RPP genes.

\section{Discussion}

\section{$T c^{r}$ bacteria are more diverse than ever thought}

In 2001, 39 genera of Gram-negative bacteria and 23 genera of Gram-positive bacteria and related genera had been described in which the mechanism of tetracycline resistance had been determined ${ }^{8)}$. In 2005, the number of $\mathrm{Tc}^{\mathrm{r}}$ bacterial genera had increased from 62 to $115^{19}$. Furthermore, in this study we found 13 genera in which tet genes had not been previously detected: identified as members of the genera Kurthia, Pediococcus, and Vagococcus in fecal isolates; Bordetella, Bradyrhizobium, and Ochrobactrum in FYM isolates; and Burkholderia, Dyella, Flexibacter, Luteibacter, Lysobacter, "Nordella", and Rhodopseudomonas in soil isolates (Table 2).

There are two reasons why bacterial genera conferring the $\mathrm{Tc}^{\mathrm{r}}$ phenotype have been found here for the first time. Firstly, we tried to isolate $\mathrm{Tc}^{\mathrm{r}}$ bacteria from various sources, such as feces, FYM, and soils. Previous studies on Tcr bacteria, on the other hand, mostly focused on enterobacterial pathogens of fecal origin, and these few studies determined the $\mathrm{Tc}^{\mathrm{r}}$ phenotype among heterotrophic bacterial flora. Secondly, we used the non-selective PTYG medium, a well- 
Table 2. Phylogenic affiliations of tetracycline-resistant bacteria isolated from different Japanese environmental samples

\begin{tabular}{|c|c|c|c|c|c|c|c|c|c|c|c|}
\hline \multirow{3}{*}{ Genus assigned } & \multicolumn{2}{|c|}{ 16S rRNA gene sequences comparison } & \multicolumn{9}{|c|}{ No. of isolates } \\
\hline & \multirow{2}{*}{$\begin{array}{l}\text { Closest relative and } \\
\text { accession number }\end{array}$} & \multirow{2}{*}{$\begin{array}{c}\text { Similarity } \\
(\%)\end{array}$} & \multirow{2}{*}{ Total } & \multicolumn{2}{|c|}{ Feces } & \multicolumn{3}{|c|}{ FYM } & \multicolumn{3}{|c|}{ Soil } \\
\hline & & & & Swine & Poultry & Swine & Poultry & Cattle & Soil $(+)^{\mathrm{a}}$ & Soil $(-)^{b}$ & F-soilc \\
\hline Acinetobacter & Acinetobacter sp. (AJ007008) & 98.6 & $2(2)$ & $2(2)$ & & & & & & & \\
\hline Afipia & A. massiliensis (AY029562) & 98.5 & $6(6)$ & & & & & & $6(6)$ & & \\
\hline \multirow[t]{3}{*}{ Alcaligenes } & Alcaligenes sp. (AY346138) & 98.8 & $6(1)$ & & $1(1)$ & & $5(0)$ & & & & \\
\hline & Alcaligenes sp. (AY628412) & 97.6 & $2(2)$ & & $2(2)$ & & & & & & \\
\hline & Alcaligenes sp. (AJ786800) & 95.8 & $1(1)$ & & & & $1(1)$ & & & & \\
\hline Bacillus & Bacillus sp. (AF169535) & 98.9 & $1(1)$ & & & $1(1)$ & & & & & \\
\hline Bordetella* & Bordetella sp. (DQ302159) & $96.1-98.0$ & $6(3)$ & & & & $6(3)$ & & & & \\
\hline \multirow{3}{*}{ Bradyrhizobium* } & B. elkanii (AY904771) & $99.1-99.8$ & $3(1)$ & & & $1(0)$ & $1(1)$ & & & $1(0)$ & \\
\hline & Bradyrhizobium sp. (DQ100058) & 100 & $1(0)$ & & & $1(0)$ & & & & & \\
\hline & Bradyrhizobium sp. (AY603956) & $99.9-100$ & $3(0)$ & & & & & & & & $3(0)$ \\
\hline \multirow[t]{3}{*}{ Burkholderia* } & B. cepacia (AY677087) & 99.4 & $10(1)$ & & & & & & $1(0)$ & & $9(1)$ \\
\hline & B. cepacia (AY769903) & 99.3 & $5(1)$ & & & & & & $3(1)$ & & $2(0)$ \\
\hline & Burkholderia sp. (DQ847125) & 98.8 & $32(15)$ & & & & & & $8(5)$ & $14(10)$ & $10(0)$ \\
\hline Clostridium & Clostridium sp. (AF443595) & 94.5 & $3(2)$ & & & & $3(2)$ & & & & \\
\hline Dyella* & D. koreensis (AY884571) & 98.3 & $5(1)$ & & & & & & & & $5(1)$ \\
\hline Enterococcus & E. faecalis (AY850358) & 100 & $60(58)$ & $22(22)$ & $38(36)$ & & & & & & \\
\hline \multirow[t]{3}{*}{ Escherichia } & E. coli (U00096) & $99.5-100$ & $43(43)$ & $13(13)$ & $29(29)$ & $1(1)$ & & & & & \\
\hline & E. coli (AM184233) & 99.4 & $9(8)$ & $1(1)$ & & $8(7)$ & & & & & \\
\hline & E. coli (AM184252) & 99.0 & $1(1)$ & $1(1)$ & & & & & & & \\
\hline Flexibacter* & Flexibacter sp. (AY238335) & $97.1-99.0$ & $19(12)$ & & & & & & $4(3)$ & $12(6)$ & $3(3)$ \\
\hline Inquilinus & I. ginsengisoli (AB245352) & 99.4 & $1(0)$ & & & & & & & & $1(0)$ \\
\hline \multirow[t]{3}{*}{ Kurthia* } & K. gibsonii (AM184261) & $96.7-99.9$ & $11(11)$ & $5(5)$ & $6(6)$ & & & & & & \\
\hline & K. gibsonii (DQ071565) & 99.1 & $1(1)$ & & $1(1)$ & & & & & & \\
\hline & K. zopfii $(\mathrm{X} 70321)$ & 99.5 & $1(1)$ & & $1(1)$ & & & & & & \\
\hline \multirow[t]{2}{*}{ Luteibacter* } & L. rhizovicinus (AY162032) & 98.3 & $4(0)$ & & & & & & & & $4(0)$ \\
\hline & L. rhizovicinus (AJ580499) & 98.2 & $1(1)$ & & & & & & & & $1(1)$ \\
\hline \multirow[t]{3}{*}{ Lysobacter* } & L. antibioticus (AB019582) & 99.3 & $2(2)$ & & & & & & & $2(2)$ & \\
\hline & L. gummosus (AB161361) & 99.5 & $2(1)$ & & & & & & & $2(1)$ & \\
\hline & Lysobacter sp. (AB083480) & 100 & $1(0)$ & & & & & & & & $1(0)$ \\
\hline Nordella* & N. oligomobilis (AF370880) & 98.3 & $4(4)$ & & & & & & $4(4)$ & & \\
\hline \multirow[t]{2}{*}{ Ochrobactrum* } & O. grignonense (AJ242581) & 97.3 & $3(3)$ & & & $3(3)$ & & & & & \\
\hline & Ochrobactrum sp. (DQ305290) & 99.3 & $2(0)$ & & & & & $2(0)$ & & & \\
\hline Pandoraea & Pandoraea sp. (AF247699) & 99.8 & $1(0)$ & & & & & & & & $1(0)$ \\
\hline Pediococcus* & Pediococcus sp. (AB219053) & 99.9 & $10(7)$ & & $10(7)$ & & & & & & \\
\hline Proteus & P. mirabilis (AF008582) & $99.8-100$ & $8(8)$ & $7(7)$ & $1(1)$ & & & & & & \\
\hline & Proteus sp. (DQ512963) & 98.2 & $1(1)$ & & $1(1)$ & & & & & & \\
\hline Providencia & P. heimbachae (AM040490) & 99.5 & $6(4)$ & & $6(5)$ & & & & & & \\
\hline Rhodopseudomonas* & Rhodopseudomonas sp. (AB251406) & 97.0 & $1(1)$ & & & & & & $1(1)$ & & \\
\hline Serratia & S. marcescens (AJ302156) & $99.5-100$ & $30(22)$ & $13(5)$ & $1(0)$ & & & $16(16)$ & & & \\
\hline & S. marcescens (AY514435) & 99.1 & $5(4)$ & & & & & & $4(4)$ & & $1(0)$ \\
\hline & Serratia sp. (AM396490) & 99.0 & $3(2)$ & $3(2)$ & & & & & & & \\
\hline Staphylococcus & S. epidermidis (СР000029) & $99.4-99.8$ & $6(4)$ & $1(1)$ & & & & & $5(3)$ & & \\
\hline & S. equorum (AM237374) & $99.6-99.8$ & $3(0)$ & & & & $3(0)$ & & & & \\
\hline & S. saprophyticus (AP008934) & 100 & $12(7)$ & $6(2)$ & $3(3)$ & $3(2)$ & & & & & \\
\hline & S. xylosus (AF515587) & $99.0-99.5$ & $7(3)$ & & & $3(0)$ & $4(3)$ & & & & \\
\hline Streptomyces & S. olivaceus (AB184735) & 99.5 & $1(0)$ & & & & & & & & $1(0)$ \\
\hline Vagococcus* & V. fluvialis (Y18098) & 100 & $1(1)$ & & $1(1)$ & & & & & & \\
\hline Variovorax & V. paradoxus (DQ256487) & 99.1 & $1(0)$ & & & & & & & $1(0)$ & \\
\hline & Variovorax sp. (AY689027) & 100 & $2(0)$ & & & & & & & & $2(0)$ \\
\hline Total & & & $350(249)$ & $74(61)$ & $102(96)$ & $21(14)$ & $23(10)$ & $18(16)$ & $36(27)$ & $32(19)$ & $44(6)$ \\
\hline
\end{tabular}

Numbers in the table show numbers of isolates belonging to the respective genus after RFLP analysis.

a: Soil treated with FYM. b: Soil not treated with FYM. ${ }^{c}$ : Forest soil.

( ): the number of isolates having tet genes, *: genus in which tet genes were found for the first time. 
Table 3. Tetracycline efflux genes detected in bacterial isolates

\begin{tabular}{lll}
\hline & \multicolumn{3}{c}{ Efflux genes } \\
\cline { 2 - 4 } Genus assigned & $\begin{array}{c}\text { Number of } \\
\text { isolates }\end{array}$ & Origin \\
\hline
\end{tabular}

\begin{tabular}{|c|c|c|c|}
\hline $\operatorname{tet}(\mathrm{A})$ & $\begin{array}{l}\text { Alcaligenes } \\
\text { Escherichia }\end{array}$ & $\begin{array}{l}2 \\
4\end{array}$ & $\begin{array}{l}\text { Poultry feces } \\
\text { Swine feces }\end{array}$ \\
\hline \multirow[t]{21}{*}{$\operatorname{tet}(\mathrm{B})$} & Afipia & 6 & Poultry FYM applied soil \\
\hline & Alcaligenes & 1 & Poultry FYM \\
\hline & Bordetella & 1 & Poultry FYM \\
\hline & Bradyrhizobium & 1 & Poultry FYM \\
\hline & Burkholderia & 1 & Swine FYM applied soil \\
\hline & & 1 & Forest soil \\
\hline & Enterococcus & 2 & Poultry feces \\
\hline & Escherichia & 4 & Swine feces \\
\hline & & 2 & Swine FYM \\
\hline & Flexibacter & 3 & Forest soil \\
\hline & Kurthia & 2 & Swine feces \\
\hline & & 3 & Poultry feces \\
\hline & Ochrobactrum & 3 & Swine FYM \\
\hline & Pediococcus & 2 & Poultry feces \\
\hline & Providencia & 3 & Poultry feces \\
\hline & Serratia & 5 & Swine feces \\
\hline & & 2 & Cattle FYM \\
\hline & Staphylococcus & 1 & Swine feces \\
\hline & & 1 & Poultry FYM \\
\hline & & 3 & Poultry FYM applied soil \\
\hline & Vagococcus & 1 & Poultry feces \\
\hline
\end{tabular}

tet(C) Escherichia Staphylococcus

tet $(\mathrm{G}) \quad$ Flexibacter Rhodopseudomonas

tet $(\mathrm{H})$ Alcaligenes

Enterococcus

Escherichia

Kurthia

Pediococcus

Proteus

Serratia

Staphylococcus

tet $(\mathrm{J})$ Burkholderia

Escherichia

Lysobacter

Nordella

Proteus

Serratia

Pediococcus

Providencia

Serratia

tet $(\mathrm{Y})$ Acinetobacter

Dyella

Luteibacter

tet(Z) Burkholderia

Serratia
Poultry feces

Poultry feces

Swine feces

FYM not applied soil

Poultry FYM applied soil

Poultry feces

Swine feces

Swine feces

Poultry feces

Poultry feces

Poultry feces

Swine feces

Poultry feces

Poultry feces

Swine FYM applied soil

FYM not applied soil

Swine FYM

FYM not applied soil

Poultry FYM applied soil

Swine feces

Swine feces

Poultry feces

Poultry feces

Cattle FYM

Swine feces

Forest soil

Forest soil

Poultry FYM applied soil

FYM not applied soil

Cattle FYM

Poultry FYM applied soil
Table 4. Tetracycline RPP genes detected in bacterial isolates

\begin{tabular}{|c|c|c|c|}
\hline \multirow{2}{*}{ Gene } & \multicolumn{3}{|c|}{ RPP genes } \\
\hline & Genus assigned $^{a}$ & $\begin{array}{c}\text { Number of } \\
\text { isolates }\end{array}$ & Origin \\
\hline \multirow[t]{12}{*}{$\operatorname{tet}(\mathrm{M})$} & Bacillus & 1 & Swine FYM \\
\hline & Burkholderia & 1 & FYM not applied soil \\
\hline & Enterococcus & 20 & Swine feces \\
\hline & & 30 & Poultry feces \\
\hline & Escherichia & 1 & Swine feces \\
\hline & & 7 & Poultry feces \\
\hline & & 1 & Swine FYM \\
\hline & Flexibacter & 2 & FYM not applied soil \\
\hline & Kurthia & 3 & Swine feces \\
\hline & & 5 & Poultry feces \\
\hline & Proteus & 1 & Poultry feces \\
\hline & Staphylococcus & 2 & Swine FYM \\
\hline \multirow[t]{2}{*}{$\operatorname{tet}(\mathrm{O})$} & Enterococcus & 2 & Poultry feces \\
\hline & Pediococcus & 1 & Poultry feces \\
\hline \multirow[t]{2}{*}{$\operatorname{otr}(\mathrm{A})$} & Burkholderia & 1 & FYM not applied soil \\
\hline & Serratia & 1 & Swine FYM applied soil \\
\hline \multirow[t]{4}{*}{$\operatorname{tet}(\mathrm{Q})$} & Enterococcus & 1 & Swine feces \\
\hline & Flexibacter & 1 & Poultry FYM applied soil \\
\hline & Providencia & 1 & Poultry feces \\
\hline & Staphylococcus & 1 & Swine feces \\
\hline \multirow[t]{2}{*}{$\operatorname{tet}(\mathrm{S})$} & Clostridium & 1 & Poultry FYM \\
\hline & Escherichia & 1 & Swine FYM \\
\hline \multirow[t]{15}{*}{$\operatorname{tet}(\mathrm{W})$} & Acinetobacter & 1 & Swine feces \\
\hline & Bordetella & 1 & Poultry FYM \\
\hline & Burkholderia & 1 & FYM not applied soil \\
\hline & & 1 & Poultry FYM \\
\hline & Clostridium & 2 & Poultry FYM \\
\hline & Enterococcus & 4 & Poultry feces \\
\hline & Escherichia & 2 & Swine feces \\
\hline & & 2 & Swine FYM \\
\hline & Flexibacter & 3 & FYM not applied soil \\
\hline & & 2 & Swine FYM applied soil \\
\hline & Nordella & 1 & Poultry FYM applied soil \\
\hline & Pediococcus & 1 & Poultry feces \\
\hline & Serratia & 1 & Swine feces \\
\hline & & 2 & Cattle FYM \\
\hline & Staphylococcus & 2 & Poultry FYM \\
\hline
\end{tabular}

${ }^{a}$ Genus was assigned based on partial 16S rRNA gene sequences.

known low-nutrient medium used for isolating oligotrophic soil bacteria. Although it is surprising that $\mathrm{Tc}^{\mathrm{r}}$ bacteria were isolated from fecal and FYM samples using the PTYG medium, our results show that PTYG medium is well applicable to isolating oligotrophic bacteria, even from fecal and FYM samples.

${ }^{a}$ Genus was assigned based on partial 16S rRNA gene sequences. 
$\mathrm{Tc}^{\mathrm{r}}$ bacterial isolates seem to be more diverse than our study would suggest, because there is a report that CFU counts were about 10 times higher when a gellan gum-based medium rather than agar-based medium was used with freshwater sediment samples ${ }^{11)}$. If we were to use, for example, a gellan gum-based medium, we may obtain more diverse $\mathrm{Tc}^{\mathrm{r}}$ isolates. Further studies are needed to isolate culturable bacteria.

\section{A number of tet genes newly identified in Tcr bacterial genera}

In this study, we found a number of tet genes in the documented $\mathrm{Tc}^{\mathrm{r}}$ bacterial genera as well as in some genera not previously identified as being tetracycline resistant. Of the 115 previously documented $\mathrm{Tc}^{\mathrm{r}}$ bacterial genera, 11 were found in this study. In Gram-negative bacteria, we found the $\operatorname{tet}(\mathrm{Y})$ and tet $(\mathrm{W})$ genes in Acinetobacter, the tet $(\mathrm{B})$ gene in Afipia, the tet $(\mathrm{B})$ and $\operatorname{tet}(\mathrm{H})$ genes in Alcaligenes, the $\operatorname{tet}(\mathrm{W})$ gene in Escherichia, the tet $(\mathrm{H})$ and $\operatorname{tet}(\mathrm{M})$ genes in Proteus, the tet $(\mathrm{J})$ and $\operatorname{tet}(\mathrm{Q})$ genes in Providencia, and the tet $(\mathrm{H}), \operatorname{tet}(\mathrm{J})$, tet $(\mathrm{Z})$, otr $(\mathrm{A})$, and $\operatorname{tet}(\mathrm{W})$ genes in Serratia. In Gram-positive bacteria, we found the $\operatorname{tet}(\mathrm{S})$ and $\operatorname{tet}(\mathrm{W})$ genes in Clostridium, the $\operatorname{tet}(\mathrm{B}), \operatorname{tet}(\mathrm{H}), \operatorname{tet}(\mathrm{Q})$, and $\operatorname{tet}(\mathrm{W})$ genes in Enterococcus, and the $\operatorname{tet}(\mathrm{B}), \operatorname{tet}(\mathrm{C}), \operatorname{tet}(\mathrm{H})$, and tet $(\mathrm{Q})$ genes in Staphylococcus. These combinations are new, although a variety of tet genes have been reported in these species up to now.

In addition, 13 genera were found for the first time in this study as carrying tet genes. In Gram-negative bacteria, the Bordetella isolates contained tet(B) or tet $(\mathrm{W})$, Bradyrhizobium isolate contained tet(B), Burkholderia isolates contained tet $(\mathrm{B}), \operatorname{tet}(\mathrm{J}), \operatorname{tet}(\mathrm{Z}), \operatorname{tet}(\mathrm{M}), \operatorname{tet}(\mathrm{W}), \operatorname{or} \operatorname{otr}(\mathrm{A})$, Dyella isolate contained tet $(\mathrm{Y})$, Flexibacter isolates contained 1 of 5 tet genes $(\operatorname{tet}(\mathrm{B}), \operatorname{tet}(\mathrm{G}), \operatorname{tet}(\mathrm{M}), \operatorname{tet}(\mathrm{Q})$, or tet $(\mathrm{W}))$, Luteibacter isolate contained tet $(\mathrm{Y})$, Lysobacter isolates contained tet $(\mathrm{B})$ or tet $(\mathrm{J})$, "Nordella" isolates contained tet $(\mathrm{J})$ or tet $(\mathrm{W})$, Ochrobactrum isolates contained tet(B), and Rhodopseudomonas isolate contained tet $(\mathrm{G})$. In Gram-positive bacteria, the Kurthia isolates contained 1 of 3 tet genes (tet $(\mathrm{B})$, tet $(\mathrm{H})$, or tet $(\mathrm{M}))$, Pediococcus isolates contained tet $(\mathrm{B})$, tet $(\mathrm{H}), \operatorname{tet}(\mathrm{J})$, tet $(\mathrm{O})$, or tet $(\mathrm{W})$, and Vagococcus isolate contained tet $(\mathrm{B})$.

One reason for these new findings is that the scale of the experiment on the tet gene in the agricultural environment was small, and there is a possibility that the tet gene exists in various bacteria.

\section{Broad or narrow host range of the tet genes}

The tet genes are found in a variety of bacteria isolated from humans, animals, and the environment. The majority of the tet genes are associated with either conjugative or mobilizable elements which may partially explain their wide distribution among bacterial species. The Gram-negative tet efflux genes are found as transposons inserted into plasmids of a diverse group from plasmids of a variety of incompatibility groups. Gram-positive efflux genes are associated with small plasmids. The ribosomal protection genes tet $(\mathrm{S})$ and $\operatorname{tet}(\mathrm{O})$ can be found on conjugative plasmids, or in the chromosome, where they are not self-mobile. The $\operatorname{tet}(\mathrm{M})$ and $\operatorname{tet}(\mathrm{Q})$ genes are generally associated with conjugative chromosomal elements, which code for their own transfer ${ }^{8}$.

The tet(B) gene has the widest host range of any Gramnegative bacterium and often presents on a conjugative plasmid $^{8)}$. Also in our study, tet(B) was detected in a wide range of bacterial genera isolated from animal feces, FYM, and soil (Table 3$)$. Similarly, $\operatorname{tet}(\mathrm{M}), \operatorname{tet}(\mathrm{W})$, and tet $(\mathrm{J})$ were ubiquitously detected in various environments. It has been well established that bacteria carrying tet $(\mathrm{M})$ and $\operatorname{tet}(\mathrm{W})$ belong to various genera ${ }^{19}$, and are often associated with conjugative transposons. In the case of the tet $(\mathrm{J})$ gene, we do not yet have enough information, with only one report of its detection in Proteus $^{8}$. The reason why tet(B), tet(M), and $\operatorname{tet}(\mathrm{W})$ were detected in numerous genera is thought to be because of such conjugative determinants ${ }^{23)}$.

On the other hand, some tet genes were detected only in limited environments. In this study, $\operatorname{tet}(\mathrm{A}), \operatorname{tet}(\mathrm{C}), \operatorname{tet}(\mathrm{H})$, and $\operatorname{tet}(\mathrm{O})$ were only detected in animal feces. These four genes have been frequently detected in isolates associated with the gastrointestinal tract of pigs or other farm animals ${ }^{1,6,10,13)}$. One reason why these tet genes were distributed in limited hosts is their lower mobility. Another explanation is that these tet genes are less well studied than other wide host range tet genes. Indeed, tet $(\mathrm{H})$ was detected in animals and fish farm water ${ }^{16)}$. A recent report also detected $\operatorname{tet}(\mathrm{H})$ in manure-applied soil ${ }^{21)}$.

In this study, we isolated 350 tetracycline-resistant bacteria from 19 wide-ranging types of environmental samples and investigated the distribution of 19 tet genes. Our results suggested that even usually nonpathogenic and nonclinical bacteria had tetracycline resistance genes at a high rate and that $\mathrm{Tc}^{\mathrm{r}}$ bacteria are more diverse than previously thought. The risk of antibiotic resistance genes diffusing from animal production sites to various environments is a serious public health problem. Further studies will be needed for a detailed understanding of gene transfer in various environments. Such knowledge may be useful for risk assessment in the future. 


\section{Acknowledgements}

We sincerely thank Toshiaki Nakajima-Kambe and Nobuhiko Nomura of the University of Tsukuba, Takeshi Fujii of the National Institute for Agro-Environmental Sciences (NIAES), and Kiyoshi Tajima of the National Institute of Livestock and Grassland Science (NILGS) for their advice and suggestions.

\section{References}

1) Aarestrup, F.M., Y. Agersø, P. Gerner-Smidt, M. Madsen and L.B. Jensen. 2000. Comparison of antimicrobial resistance phenotypes and resistance genes in Enterococcus faecalis and Enterococcus faecium from humans in the community, broilers, and pigs in Denmark. Diagn. Microb. Infect. Dis. 37: 127-137.

2) Agersø, Y., G. Sengeløv and L.B. Jensen. 2004. Development of a rapid method for direct detection of tet $(\mathrm{M})$ genes in soil from Danish farmland. Environ. Int. 30: 117-122.

3) Aminov, R.I., J.C. Chee-Sanford, N. Garrigues, B. Teferedegne, I.J. Krapac, B.A. White and R.I. Mackie. 2002. Development, validation, and application of PCR primers for detection of tetracycline efflux genes of Gram-negative bacteria. Appl. Environ. Microbiol. 68: 1786-1793.

4) Aminov, R.I., N. Garrigues-Jeanjean and R.I. Mackie. 2001. Molecular ecology of tetracycline resistance: Development and validation of primers for detection of tetracycline resistance genes encoding ribosomal protection proteins. Appl. Environ. Microbiol. 67: 22-32.

5) Ardic, N., M. Ozyurt, B. Sareyyupoglu and T. Haznedaroglu. 2005. Investigation of erythromycin and tetracycline resistance genes in methicillin-resistant staphylococci. Int. J. Antimicrob. Agents 26: 213-218.

6) Blake, D.P., R.W. Humphry, K.P. Scott, K. Hillman, D.R. Fenlon and J.C. Low. 2003. Influence of tetracycline exposure on tetracycline resistance and the carriage of tetracycline resistance genes within commensal Escherichia coli populations. J. Appl. Microbiol. 94: 1087-1097.

7) Chee-Sanford, J.C., R.I. Aminov, I.J. Krapac, N. Garrigues-Jeanjean and R.I. Mackie. 2001. Occurrence and diversity of tetracycline resistance genes in lagoons and groundwater underlying two swine production facilities. Appl. Environ. Microbiol. 67: 14941502.

8) Chopra, I. and M.C. Roberts. 2001. Tetracycline antibiotics: Mode of action, applications, molecular biology, and epidemiology of bacterial resistance. Microbiol. Mol. Biol. Rev. 65: 232260.

9) Furushita, M., T. Shiba, T. Maeda, M. Yahata, A. Kaneoka, Y.
Takahashi, K. Torii, T. Hasegawa and M. Ohta. 2003. Similarity of tetracycline resistance genes isolated from fish farm bacteria to those from clinical isolates. Appl. Environ. Microbiol. 69: 53365342 .

10) Hansen, L.M., P.C. Blanchard and D.C. Hirsh. 1996. Distribution of $\operatorname{tet}(\mathrm{H})$ among Pasteurella isolates from the United States and Canada. Antimicrob. Agents. Chemother. 40: 1558-1560.

11) Kamagata, Y. and H. Tamaki. 2005. Cultivation of uncultured fastidious microbes. Microbes. Environ. 20: 85-91.

12) Kobashi, Y., A. Hasebe and M. Nishio. 2005. Antibiotic-resistant bacteria from feces of livestock, farmyard manure, and farmland in Japan-Case report-. Microbes. Environ. 20: 53-60.

13) Lanz, R., P. Kuhnert and P. Boerlin. 2003. Antimicrobial resistance and resistance gene determinants in clinical Escherichia coli from different animal species in Switzerland. Vet. Microbiol. 91: 73-84.

14) Maniatis, T., E.F. Frintsch and J. Sambrook. 1982. Molecular cloning: A laboratory manual, Cold Spring Harbor Laboratory, Cold Spring Harbor, N.Y.

15) Marchesi, J.R., T. Sato, A.J. Weightman, T.A. Martin, J.C. Fry, S.J. Hiom and W.G. Wade. 1998. Design and evaluation of useful bacterium-specific PCR primers that amplify genes coding for bacterial 16S rRNA. Appl. Environ. Microbiol. 64: 795-799.

16) Miranda, C.D., C. Kehrenberg, C. Ulep, S. Schwarz and M.C. Roberts. 2003. Diversity of tetracycline resistance genes in bacteria from Chilean salmon farms. Antimicrob. Agents Chemother. 47: 883-888.

17) Moyer, C.L., J.M. Tiedje, F.C. Dobbs and D.M. Karl. 1996. A computer-simulated restriction fragment length polymorphism analysis of bacterial small-subunit rRNA genes: efficacy of selected tetrameric restriction enzymes for studies of microbial diversity in nature. Appl. Environ. Microbiol. 62: 2501-2507.

18) Petersen, A. and A. Dalsgaard. 2003. Species composition and antimicrobial resistance genes of Enterococcus spp., isolated from integrated and traditional fish farms in Thailand. Environ. Microbiol. 5: 395-402.

19) Roberts, M.C. 2005. Update on acquired tetracycline resistance genes. FEMS Microbiol. Lett. 245: 195-203.

20) Rogers, S.O. and A.J. Bendich. 1985. Extraction of DNA from milligram amounts of fresh, herbarium and mummified plant tissues. Plant Mol. Biol. 5: 69-76.

21) Schmitt, H., K. Stoob, G. Hamscher, E. Smit and W. Seinen. 2006. Tetracyclines and tetracycline resistance in agricultural soils: microcosm and field studies. Microb. Ecol. 51: 267-276.

22) Sebgeløv, G., Y. Agersø, B. Halling-Sørensen, S.B. Baloda, J.S. Andersen and L.B. Jensen. 2003. Bacterial antibiotic resistance levels in Danish farmland as a result of treatment with pig manure slurry. Env. Int. 28: 587-595.

23) Taylor, D.E. and A. Chau. 1996. Tetracycline resistance mediated by ribosomal protection. Antimicrob. Agents. Chemother. 40: 1-5. 